\title{
MULTI-FREQUENCY RADIO OBSERVATIONS OF SPIRAL GALAXIES AND THEIR INTERPRETATION
}

\author{
M. KRAUSE \\ Max-Planck-Institut für Radioastronomie \\ Auf dem Hügel 69 \\ 5300 Bonn 1 \\ F.R.G.
}

\begin{abstract}
After a brief historical summary of radio observations of spiral galaxies I review the methods of analyzing radio polarization data in view of the magnetic field. Special attention is drawn to the Faraday rotation and depolarization effects and to the identification of the largescale magnetic field structure. The present observational results and open questions are discussed in terms of the predictions of the dynamo theory and prospects on future work are given.
\end{abstract}

\section{Introduction}

The magnetic field structure of spiral galaxies can be best determined so far by radio observations of total and linearly polarized continuum emission. There has been a major increase in sensitivity since the first detection of linear polarization in a spiral galaxy (M51) by Mathewson et al. (1972). These measurements proved for the first time that a significant amount of the total intensity of galaxies is due to synchrotron radiation and hence the existence of magnetic fields in spiral galaxies.

They were followed by polarization observations also carried out with the Westerbork Synthesis Radio Telescope (WSRT) of M81 and M51 by Segalovitz et al. (1976) and by those of M31 made with the Effelsberg 100-m telescope (Beck et al., 1978). However, they detected polarized intensity only in small individual parts of these galaxies. First complete maps of linear polarization of a spiral galaxy were obtained with the Effelsberg 100-m telescope of M31 and M33 (Beck, 1979). Up to now unique polarization data from about a dozen spiral galaxies have been collected with the $100-\mathrm{m}$ radio telescope (see Table 1).

Since 1980 numerous sources have been observed in linear polarization with the Very Large Array (VLA). Only in 1987, first complete maps of spiral galaxies (M81 and IC 342) have been obtained with this telescope in order to derive the large-scale magnetic field structure (Krause et al., $1987 \mathrm{a}, \mathrm{b})$. Since that time many galaxies have been observed successfully in linear polarization with the VLA (see Table 1).

Recently the 64-m Parkes telescope was used to study the magnetic field structure in southern galaxies (see Table 1), starting with observations of the SMC (Haynes et al., 1986). The completion of the Australia 

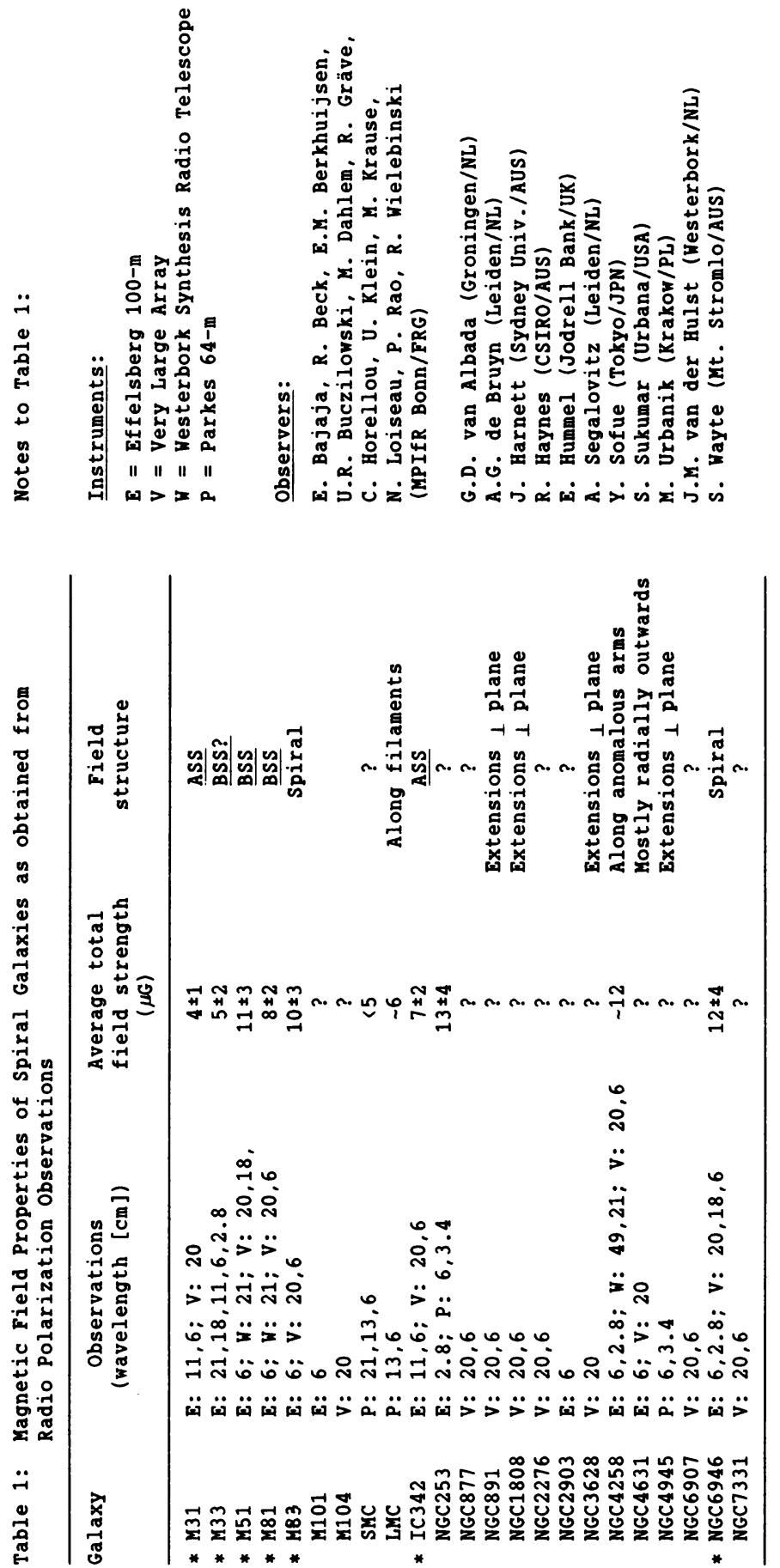
Telescope (AT) will lead to a significant progress in observing southern spiral galaxies in more detail and with better sensitivity and thus give the opportunity to increase the number of spiral galaxies being suitable for an analysis of the magnetic field.

\section{Methods of Analyzing Radio Polarization Data}

\subsection{In view of the magnetic field in general}

Radio continuum observations of total and linearly polarized intensity enables to determine the degree of linear polarization $p$ and the orientation of the electric vector $\chi$. The intrinsic electric vector $\chi_{E}$ of the linearly polarized emission is perpendicular to the magnetic field component $\left(B_{\perp}\right)$ projected onto the plane of the sky and gives the orientation of $B_{\perp}$. The observed electric vector must be corrected for Faraday rotation. The rotation measure $\mathrm{RM}$ itself can be determined by observations at two frequencies (or more to solve its ambiguity). It is proportional to $\int n_{e} B_{\|} d \ell$, hence giving the direction of $B_{\|}$(i.e. the magnetic field component parallel to the line of sight) and - if the thermal electron density $n_{e}$ and pathlength $\ell$ is known - its strength.

The strength of $B_{\perp}$ can be estimated from the nonthermal (synchrotron) radio emission with the assumption of pressure equilibrium (or energy equipartition) between cosmic rays and the magnetic field (see e.g. Krause et al., 1984). If $B_{\perp}$ fluctuates along the line of sight, only an upper limit can be derived from the observations (see Beck, 1986). Presuming that the strongest magnetic field component lies within the disk of the galaxy the total field strength $\left(B_{t}\right)$ in the disk and the strength of $B_{\|}$can be derived. The latter should be consistent with the value obtained from the rotation measure as described above.

The observed degree of the nonthermal linear polarization $p_{n}$ can be used to estimate the degree of uniformity $B_{u} / B_{t}$ (see Segalovitz et al., 1976). $B_{u}$ is the magnetic field component with a uniform orientation within the beam area and depends on the resolution of the observation. The observed rotation measure is a superposition of the intrinsic (in the source of the radiation) rotation measure $R_{i}$ and the foreground rotation measure $R M_{f g}$. As has been mentioned already by Burn (1966), $R M_{i}$ is only proportional to $\lambda^{2}$ for $R M_{i} \lambda^{2} \leqslant 1 \mathrm{rad}$, due to the mixture of emitting and Faraday rotating media which produce Faraday dispersion and differential Faraday rotation. Table 2 shows the upper limits of rotation measure values which can be interpreted as $\mathrm{RM}_{i}$ obeying the $\lambda^{2}-$ law. If the observed rotation measure exceeds the values listed in Table 2, it cannot originate in the source alone (as $R M_{i}$ ) but must be at least partly due to the foreground.

The foreground rotation measure is composed of the rotation measure within the Galaxy and that of the intergalactic space. As long as there is no cluster of galaxies along the line of sight, the intergalactic RM can be neglected for galaxies with small redshift $z$ as it is proportional to $(1+\mathrm{z})^{\beta}$ with $\beta \simeq 0.9 \pm 0.6$ (Welter et al., 1984).

The foreground rotation in the Galaxy generally has to be taken into account. It rotates the electric vector when passing through it (following the $\lambda^{2}$-law even when its value exceeds those listed in Table 2). In 
Table 2.

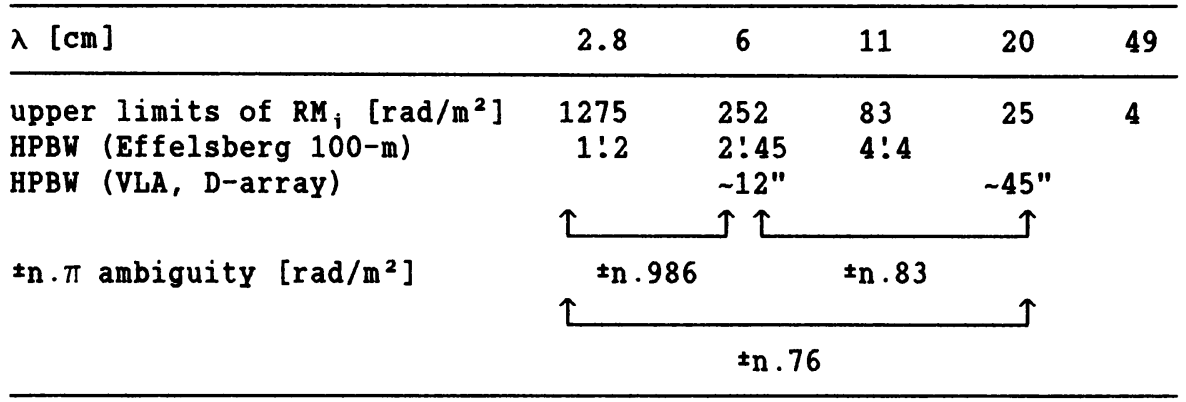

principle it also can depolarize the radiation if the fluctuations of $\mathrm{RM}_{\mathrm{f}}$ are small compared to the beam size and the pathlength through the Galaxy. However, as the depolarization is proportional to $e^{-2 \sigma_{R M} \lambda^{4}}$ (Burn, 1966) and $\sigma_{R M}$ is about $10 \mathrm{rad}^{2} / \mathrm{m}^{4}$ in most parts of the Galaxy (Simonetti and Cordes, 1986), it is only $3 \%$ even at $\lambda 20 \mathrm{~cm}$. Hence, in most cases the external Faraday dispersion can be neglected.

Main depolarization occurs by internal Faraday dispersion (Burn, 1966; discussion in e.g. Krause et al., 1989a) due to the turbulent magnetic field component $B_{r}$ and to $B_{u}$ (which should be rather called "differential Faraday rotation") in the emitting source and a dispersion of the observed E-vectors within the solid angle of the telescope's beam (that is what I call "beam depolarization"). In this definition the beam depolarization is wavelength independent and only contingent on the resolution of the observations.

Table 2 gives the resolution at different wavelengths for the Effelsberg $100-m$ telescope and the VLA in D-array, and the $\pm n . \pi$ ambiguities when determining the rotation measure only from observations at 2 different wavelengths. Bearing in mind that the intensity of synchrotron radiation decreases strongly with increasing frequency, observations at $\lambda 2.8 \mathrm{~cm}$ and $\lambda 6 \mathrm{~cm}$ with the Effelsberg $100-\mathrm{m}$ telescope should be combined with VLA observations at $\lambda 20 \mathrm{~cm}$ and $\lambda 6 \mathrm{~cm}$ according to the size and strength of the source. Observations at higher wavelengths than $\lambda 20 \mathrm{~cm}$ are difficult as internal depolarization effects increase strongly and $R M_{i}$ is no longer proportional to $\lambda^{2}$ for values larger than $25 \mathrm{rad} / \mathrm{m}^{2}$. (The values of $R M_{i}$ found up to now in M51, M81 and IC 342 are in the range of $10-20 \mathrm{rad} / \mathrm{m}^{2}$.) Future observations should be expanded to higher frequencies and higher resolution without decreasing sensitivity.

2.2 In view of the large-scale magnetic field as predicted by dynamo theory

The uniform magnetic field component $B_{u}$ in the disk of spiral galaxies is thought to be amplified by the action of a dynamo. Following present calculations the dynamo can most easily generate an axisymmetric-spiral structure (ASS) in its ground mode $m=0$ and a bisymmetricspiral structure (BSS) in the secondly excited mode $m=1$ (see Fig. 1). 
Both field structures have already been observed. They can be distinguished by the variation of the rotation measure with azimuthal angle $\theta_{0}$ in the plane of the galaxy: In the case of a BSS field structure the RM variation is doubly-periodical (Tosa and Fujimoto, 1978; Krause et al., $1989 \mathrm{~b})$ whereas in the case of an ASS the RM variation is singly-periodical (Krause et al., 1989a) (see Fig. 1). This analysis assumes a constant density $n_{e}$ of thermal electrons, that the magnetic field lines follow spirals with constant pitch angles and that the magnetic field strength is constant in the case of an ASS field and varies as $B\left(\theta_{0}\right)=B_{u} \cos \left(\theta_{0}-\mu\right)$ in the case of a BSS field ( $\mu$ is the position angle of the magnetic spiral at a certain radius in the plane of the galaxy).

In the case of an ASS field the zero level of the fit on the RM variation results in the foreground rotation measure $\mathrm{RM}_{\mathrm{fg}}$ and the phase of the fit gives the pitch angle $\psi$ of the magnetic field lines. In the case of a BSS magnetic field structure the fit parameters 'phase' and 'zerolevel' depend on the physical parameters $\mathrm{RM}_{\mathrm{fg}}, \psi$ and on the position angle $\mu$ (see equation in Fig. $1 \mathrm{~b}$ ).

The amplitude of the fit is in both cases proportional to the strength of $B_{u}$. It should be compared with the value of $B_{u}$ estimated from the nonthermal emission and the degree of linear polarization as explained above. Further cross-checks should be done for the foreground rotation measure and the pitch angle of the magnetic field spiral. For $R_{\mathbf{f g}}$ we get independent information from background sources and in some cases from depolarization arguments. The pitch angle $\psi$ as derived by the RM variation should be in agreement with the orientation of $B_{\perp}$ evaluated from the observed electric vectors (see above).

Only if all derived values fit as pieces of one puzzle it may be said that a field structure is ASS or BSS. However, many idealizing assumptions entered into the model (as e.g. the variation of $B$ with $\theta_{0}$ ), and the dynamo equations themselves are only calculated linearly and for idea-

a)

Axisymmetric Spiral Structure
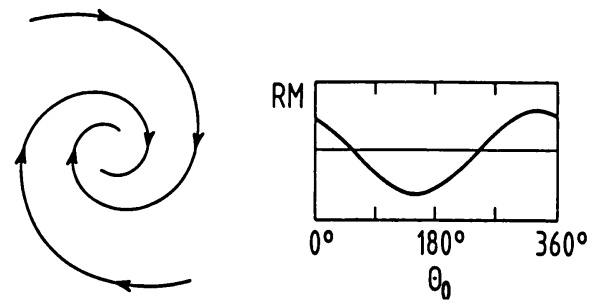

$R M\left(\theta_{0}\right)=A \cos \left(\theta_{0}-\psi\right)+R M_{f g}$ b)

Bisymmetric Spiral Structure

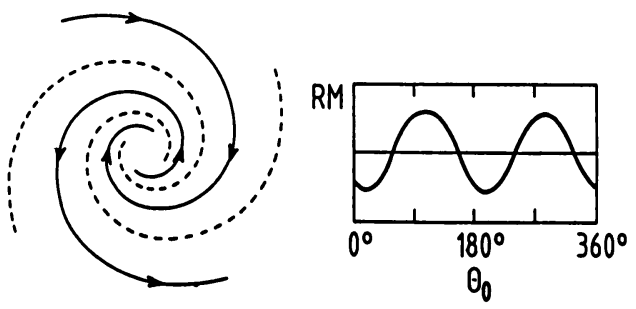

$R M\left(\theta_{0}\right)=A \cos \left(2 \theta_{0}-\psi-\mu\right)+A \cos (\mu-\psi)+R M_{f g}$

Figure 1. Axisymmetric and bisymmetric magnetic field configurations within the galactic disk (face-on view) and their rotation measure variations in the plane of the galaxy. The dots in Fig. 1b indicate the neutral lines. 
lized galaxies (e.g. with axisymmetric gas distribution). Hence it cannot be expected that the magnetic field structure of all spiral galaxies can be recognized by the above simple analysis even if all observing errors could be eliminated.

\section{Results}

Numerous galaxies have been observed up to now in linear polarization. For seven of them, large nearby face-on spiral galaxies (marked by * in Table 1), the observations have been sensitive enough and with sufficient resolution to make the analysis described above in order to distinguish between an ASS and BSS magnetic field configuration. A dominant ASS field configuration has been found in M31 (Beck, 1982; Ruzmaikin et al., 1989) and IC 342 (Krause et al., 1989a), while M81 (Krause et al., 1989b), M51 (Horellou et al., this volume) and possibly M33 (Buczllowski and Beck, this volume) have a dominant BSS field configuration. The analysis for NGC 6946 (Harnett et al., 1989; Beck et al., this volume) and M83 (Sukumar et al., in prep.) did not supply a conclusive result.

Fig. 2 shows the orientation of $B_{\perp}$ in IC 342 and Fig. 3 those of M81. The RM variations of these two galaxies with the best singly-periodic fit for IC 342 and the best doubly-periodic fit for M81 in two radial ranges in the plane of each galaxy are presented in Fig. 4. The magnetic field spirals as derived from the RM variation in Fig. 4 for M81 in each ring are indicated in Fig. 3. The change of the magnetic field direction, the so-called neutral line, is found to be located in the interarm region of M81, just where the uniform field strength $B_{u}$ is highest as derived from synchrotron emission and the degree of linear polarization.

The results of all seven galaxies concerning the magnetic field can be summarized as following:

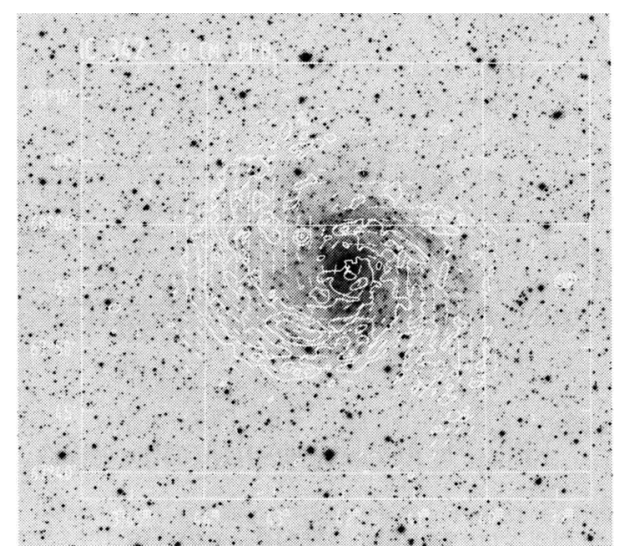

Figure 2. Contour map of the linearly polarized intensity of IC 342 , superimposed onto the red POSS plate. The HPBW is 45". Contour levels are: 0.1 , $0.3, \ldots, 1.2 \mathrm{mJy} / \mathrm{b} . \mathrm{a}$. and the r.m.s. - noise is $0.03 \mathrm{mJy} / \mathrm{b} . a$. The position angles of the vectors give the orientation of the magnetic field component $B_{\perp}$ with an angular resolution of $2: 45$ HPBW. 


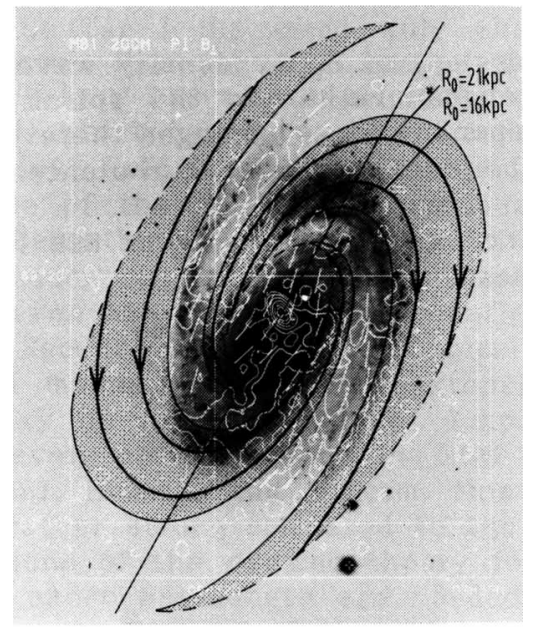

Figure 3. Contour map of the linearly polarized intensity of M81 at $1.4 \mathrm{GHz}$, superimposed onto an optical photograph made by A. Sandage with the Hale Observatories. The HPBW is $36 " \times 45 "(\mathrm{RA} \times \mathrm{DEC})$. Contour levels are: $0.1, \quad 0.2, \ldots, 1.0 \mathrm{mJy} / \mathrm{b} . \mathrm{a}$. and the r.m.s. noise is $0.05 \mathrm{mJy} / \mathrm{b} . \mathrm{a}$. The position angles of the vectors give the orientation of the magnetic field component $B_{\perp}$ perpendicular to the line of sight with an angular resolution of 2:45 HPBW. The magnetic field spirals as determined from the rotation measure distributions in Figure $4 \mathrm{a}$ are sketched. The neutral lines are located between the dotted area, namely in the interarm region.
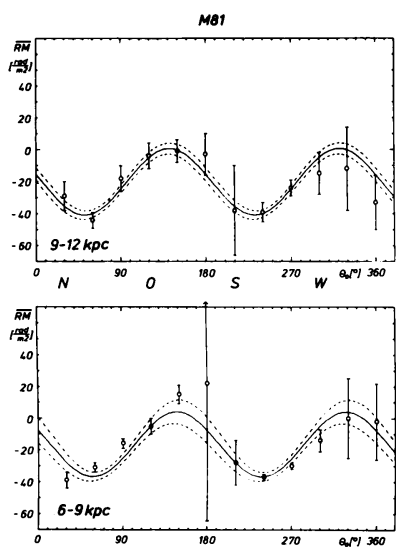

a)
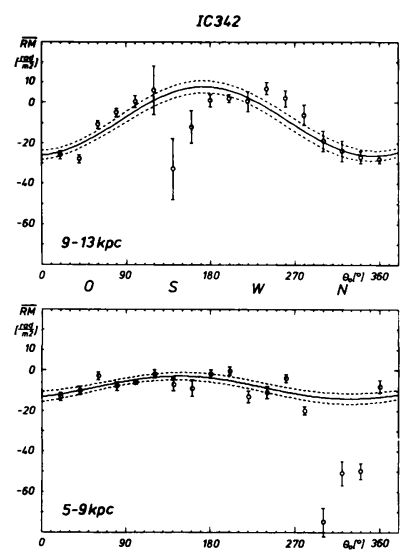

b)

Figure 4. Azimuthal rotation measure distribution of M81 and IC 342 determined between $\lambda 20$ $\mathrm{cm}$ and $\lambda 6.3 \mathrm{~cm}$ in two radial intervals in the plane of the galaxy. The full lines show the best fitted doubly-periodic (M81) resp. singly-periodic (IC 342) functions, the dashed lines their range of uncertainty.

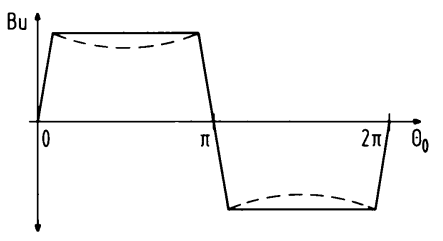

Figure 5. Sketch of $B_{u}\left(\theta_{0}\right)$ as proposed for a BSS magnetic field like in M81

- $B_{u}$ is generally orientated parallel to the optical spiral arms

- $B_{u}$ is strongest in the interarm region (i.e. between the optical spiral 
arms)

- There is no direct effect (e.g. compression) of $\mathrm{B}_{\mathrm{u}}$ by density waves As the synchrotron emission is strongest rather in the spiral arm region the random magnetic field component $B_{r}$ is stronger there than in the interarm region which may be due to higher turbulences by star formation activities in the spiral arms.

- Evidence is found for the existence of a dominant ASS or BSS magnetic field orientation in some galaxies:

$\begin{array}{lll}\frac{\text { ASS }}{\text { M31 }} & \text { BSS } & \text { inconclusive } \\ \text { IC 342 } & \text { M81 } & \text { M83 } \\ & \text { M51 } & \text { NGC 6946 } \\ & \text { M33? } & \end{array}$

\section{Future Work}

As has been mentioned in Section 3 the RM analysis of NGC 6946 and M83 give no conclusive result. It should be emphasized that this is not a problem of the quality of the observations. Moreover, the orientations of $B_{\perp}$ look quite regular and not unusual in these galaxies. Hence we have to look for possible reasons for the incompatibility of the observations with the current models (either the dynamo theory itself as calculated up to now or the way of data analysis). Most probably one or more of the idealizing assumptions given in Section 2.2 is not fulfilled as to say

- $B_{u}\left(\theta_{0}\right) \neq$ const in the case of an ASS field

- $B_{u}\left(\theta_{0}\right) \neq B_{u} \cos \left(\theta_{0}-\mu\right)$ in the case of a BSS field

Observations at least of $M 81$ indicate that the direction of $B_{u}$ in $a$ BSS configuration changes much faster near to the neutral line than sinusoidally - as assumed in the model - because the highest uniform field strength is just found near the neutral line. Higher turbulences may lower $B_{u}$ in the optical spiral arms so that $B_{u}\left(\theta_{0}\right)$ may be better approximated by the sketch in Fig. 5.

- Thermal electron density $n_{\mathrm{e}}\left(\theta_{0}\right) \neq$ const

- $B_{u}$ is not completely parallel to the plane of the galaxy as may be the case in parts of IC 342 (Krause et al., 1989a) and M31 (Beck et al., 1989) or the plane itself is warped.

- The pitch angle of the magnetic field $\psi\left(\theta_{0}\right) \neq$ const as observed in NGC 6946 (Harnett et al., 1989).

The five points mentioned above would make the RM analysis more complicated and an ASS or BSS field more difficult to detect or to distinguish. Model calculations for $R M\left(\theta_{0}\right)$ are needed which include the possible deviations from the current assumptions mentioned above.

On the other hand, even from the theoretical point of view it is not clear whether or not only one of these two field configurations could be expected to exist. There can also be

- higher modes of the dynamo

- a superposition of different dynamo modes or

- no dominant large-scale magnetic field configuration because a dynamo cannot work effectively or the galaxy has strong local distortions. 
So far I discussed only the magnetic field in the disk of spiral galaxies. However, if the magnetic field is due to the action of a dynamo the galaxy has also a poloidal field component (together with the toroidal field) which has a component in z-direction. In the ASS configuration the poloidal field is either a dipole (called odd ASS field with respect to the plane of the galaxy) or a quadrupole (called even ASS field). The two configurations have slightly different growth rates depending on the boundary conditions. Observations of IC 342 indicate that this galaxy has an even ASS magnetic field configuration (see Krause et al., 1989a).

Recent observations of spiral galaxies seen edge-on revealed a uniform magnetic field with extensions perpendicular to the plane of the galaxy up to a distance of $4 \mathrm{kpc}$ (in NGC 3628 , Krause et al., in prep.) or even up to $8 \mathrm{kpc}$ (in NGC 891 and NGC 4631, see Hummel et al. (1988); Hummel and Dahlem, this volume). These magnetic fields seem to be rather strong compared to the field strength in the disk and the predictions of the dynamo theory for disks. Observations at high resolution and short wavelength are needed in order to avoid strong depolarization along the line of sight through the disk and hence receive information about the magnetic field within the disk simultaneously. This will hopefully clarify the relation between the observed $B_{z}$ fields in edge-on galaxies and their disk fields.

Further, the magnetic field in face-on galaxies should be observed in more detail as already started in M31 (Beck and Berkhuijsen, this volume) in order to study local deviations from the large-scale structure and their dynamical processes.

\section{Acknowledgements}

I am grateful to $R$. Beck and $R$. Wielebinski for critical comments on the manuscript and thank the Deutsche Forschungsgemeinschaft for financial support under Grant No. Wi 737/2-1.

\section{References}

Beck, R. (1979) Ph.D. Thesis, University of Bonn.

Beck, R. (1982) Astron. Astrophys. 106, 121.

Beck, R. (1986) IEEE Vol. PS 14, 740.

Beck, R., Berkhuijsen, E.M. and Wielebinski, R. (1978) Astron. Astrophys. 68, L27.

Beck, R., Loiseau, N., Hummel, E., Berkhuijsen, E.M., Gräve, R. and Wielebinski, R. (1989) Astron. Astrophys. 222, 58.

Burn, B.J. (1966) Monthly Notices Roy. Astron. Soc. 133, 67.

Harnett, J.I., Beck, R. and Buczilowski, U.R. (1989) Astron. Astrophys. 208, 32.

Haynes, R.F., Klein, U., Wielebinski, R. and Murray, J.D. (1986), Astron. Astrophys. 159, 22.

Hummel, E., Lesch, H., Wielebinski, R. and Schlickeiser, R. (1988) Astron. Astrophys. 197, L29.

Krause, M., Beck, R. and Klein, U. (1984) Astron. Astrophys. 138, 385.

Krause, M., Hummel, E. and Beck, R. (1987a) in R. Beck and R. Gräve 
(eds.), Interstellar Magnetic Fields, Springer, Heidelberg, p. 52

Krause, M., Beck, R. and Hummel, E. (1987b) in R. Beck and R. Gräve

(eds.), Interstellar Magnetic Fields, Springer, Heidelberg, p. 57.

Krause, M., Hummel, E. and Beck, R. (1989a) Astron. Astrophys. 217, 4.

Krause, M., Beck, R. and Hummel, E. (1989b) Astron. Astrophys. 217, 17.

Mathewson, D.S., van der Kruit, P.C. and Brouw, W.N. (1972) Astron.

Astrophys. 17, 468.

Ruzmaikin, A., Sokoloff, D., Shukurov, A. and Beck, R. (1989) Astron.

Astrophys., in press.

Segalovitz, A., Shane, W.W. and de Bruyn, A.G. (1976) Nature 264, 222.

Simonetti, J.H. and Cordes, J.M. (1986) Astrophys. J. 310, 160.

Tosa, M. and Fujimoto, M. (1978) Publ. Astron. Soc. Japan 30, 315.

Welter, G.L., Perry, J.J. and Kronberg, P.P. (1984) Astrophys. J. 279, 19.

SHUKUROV: A short comment: It seems that your list of future problems should be complemented by one more point, namely data processing based on rigorous statistical tests.

STIX: Is there any indication that M31 has a stronger differential rotation than M81? I ask this because we have heard this morning that strong differential rotation favours the axisymmetric dynamo mode, while the non-axisymmetric dynamo may win in a galaxy with more uniform rotation.

M. KRAUSE: No, the differential rotation (or the shear $R(\partial \Omega / \partial R)$ ) is stronger in M81 than in M31. This cannot explain why M81 has a BSS field, while M31 has an ASS field structure.

SOKOLOFF: There is no theoretical prediction for the magnetic line to be a logarithmic spiral. $\psi$ must be a function of $r$. I think it is possible to include such a dependence in your picture. Is it?

M. KRAUSE: If the pitch angle $\psi$ of the magnetic spiral changes with $r$ but is constant along the azimuthal angle, it does not change the RM analysis for one radial range as the values are averaged within the considered ring in the plane of the galaxy. If you then compare, the results for different rings you can easily recognize whether $\psi$ depends on $r$. Our observations indicate that $\psi$ might increase with $r$ in M81, and decrease in IC 342. However, due to the observation errors, the data are also compatible with $\psi(r)=$ const.

SOFUE: Can you attribute the depolarization in the arm to large RM there rather than to random field? In this context, do you see any RM variation from an arm to the interarm region?

M. KRAUSE: No, especially in the south-western part of M81 - that is where the difference in degree of polarization between arm and interarm region can be most clearly seen - we see no difference in RM. 